\title{
Fractalkine (C-X3-C motif chemokine ligand 1) as a potential biomarker for depression and anxiety in colorectal cancer patients
}

\author{
DIEGO OLIVEIRA MIRANDA ${ }^{1}$, ELEN ANATRIELLO ${ }^{1}$, LUCAS RIBEIRO AZEVEDO $^{2}$, \\ JESSICA CRISTINA SANTOS ${ }^{3}$, JESSICA FERNANDA CORREA CORDEIRO ${ }^{1}$, \\ FERNANDA MARIS PERIA ${ }^{4}$, MILENA FLÓRIA-SANTOS ${ }^{5}$ and GABRIELA PEREIRA-DA-SILVA ${ }^{5}$ \\ ${ }^{1}$ Department of Maternal-Infant Nursing and Public Health, College of Nursing, University of São Paulo, \\ Ribeirão Preto, SP 14040902; ${ }^{2}$ Department of Biology, Institute of Bioscience, Language \& Literature and Exact Science, \\ São Paulo State University, São José do Rio Preto, SP 15054000; ${ }^{3}$ Postgraduate Program in Basic and Applied Immunology; \\ ${ }^{4}$ Department of Internal Medicine, School of Medicine of Ribeirão Preto, University of São Paulo; ${ }^{5}$ Post-graduate \\ Program in Public Health Nursing, College of Nursing, University of São Paulo, Ribeirão Preto, SP 14040902, Brazil
}

Received March 1, 2017; Accepted June 7, 2017

DOI: $10.3892 /$ br.2017.937

\begin{abstract}
Fractalkine, a unique chemokine of the CX3C subfamily, is involved in the pathogenesis of different types of cancer and also in non-immune mechanisms associated with psychiatric disorders. The aim of the present study was to investigate whether there is a correlation between anxiety, depression and fractalkine serum levels in colorectal cancer (CRC) patients in different stages of antitumor therapy. Four groups of patients undergoing treatment ( $\mathrm{n}=20$ per group) were evaluated: Patients with CRC who did not undergo surgical resection of the tumor; patients who underwent resection and who did not start adjuvant therapy; patients undergoing chemotherapy for $\sim 3$ months; and patients who had completed adjuvant chemotherapy regimen for $\sim 6$ months. The control group was composed of 20 healthy volunteers free of any psychiatric or immune system disease. Depression and anxiety were evaluated using the Hospital Anxiety and Depression Scale (HADS) and serum levels of fractalkine were measured by cytometric bead array. Clinically relevant levels of anxiety and/or depression were observed in all of the CRC patients at the different stages of antitumor therapy. Elevated serum levels of fractalkine were identified in the CRC patients in the pre-surgery $(\mathrm{P}<0.001)$ and pre-chemotherapy $(\mathrm{P}<0.001)$ groups, but reduced upon chemotherapy $(\mathrm{P}<0.05)$. Furthermore, a positive correlation was observed between fractalkine levels and the HADS scores in the CRC patients at different stages of antitumor therapy. These results demonstrate a link between
\end{abstract}

Correspondence to: Dr Diego Oliveira Miranda, Department of Maternal-Infant Nursing and Public Health, College of Nursing, University of São Paulo, 3900 Avenida Bandeirantes, Ribeirão Preto, SP 14040902, Brazil

E-mail:imuno@usp.br

Key words: anxiety, depression, fractalkine, colorectal cancer fractalkine, depression and anxiety in CRC patients indicating that this chemokine is involved in the pathophysiology of these comorbidities. An improved understanding of the molecular mechanisms involved in these psychological disorders will allow the design of novel therapeutic strategies to assist in alleviating such symptoms in cancer patients. Therefore, fractalkine may present as a relevant biomarker for depression and anxiety in CRC patients.

\section{Introduction}

Fractalkine (also termed C-X3-C motif chemokine ligand 1; CX3CL1) is a unique chemokine of the CX3C subfamily. It is induced by proinflammatory cytokines and identified as a secreted chemokine that is an attractant for CX3CR1-expressing mononuclear cells, and as a membrane-anchored protein that promotes leukocyte adhesion (1).

Recently, fractalkine involvement in the tumor microenvironment has been highlighted, indicating it as a potential target of cancer immunotherapy (2). In addition, fractalkine has been considered as an important marker for cancer progression $(3,4)$ including for colorectal cancer (CRC) (5).

Fractalkine has also been involved in various non-immune mechanisms associated with psychiatric disorders, including inhibition of serotonergic neurotransmission by enhancement of GABA activity at serotonergic neurons, inhibition of glutamatergic activity in the hippocampal region, and regulation of processes of neuroplasticity, such as long term potentiation (6). These mechanisms are constantly affected in the manifestation of depression and anxiety, which are the most frequent comorbidities among CRC patients (7).

Mood disorders, such as depression and anxiety, impair adherence to treatment regimens and affect the quality of life of CRC patients. Although patient quality of life is often normalized until the end of antitumor therapy, the frequent psychological monitoring of patients at risk for depression and anxiety is highly recommended (8). Thus, it is becoming increasingly important to use chemokines as risk biomarkers 
of psychological disorders, which could guide and help clinical practice.

Evidence of a role of fractalkine in the pathophysiology of depression and anxiety combined with the presence of high levels of this chemokine in cancer patients, including CRC patients (9), indicates that it contributes to mood disorders in these individuals. In the present study, the possible correlations between fractalkine and psychological manifestations were analyzed in CRC patients at various stages of antitumor therapy.

\section{Materials and methods}

Patient selection. A sample of 80 patients was selected between October/2014 and May/2015 by convenience at the Clinical Hospital of the Faculty of Medicine of Ribeirão Preto (Ribeirão Preto, SP, Brazil). Eligible participants for the study were those diagnosed with $\mathrm{CRC},>18$-years-old and male or female. These individuals were approached in the period in which they were admitted for surgical resection of the tumor, during the consultations for chemotherapy and/or return for medical evaluation. Socio-demographic data included age, sex, education and marital status. The exclusion criteria were as follows: i) Individuals who previously received or are receiving radiotherapy or chemotherapy; ii) history of chronic inflammatory or autoimmune diseases, active infectious diseases, kidney disease or diabetes; iii) use of immunosuppressive drugs; iv) patients diagnosed with schizoaffective disorder, bipolar disorder or panic disorder; and v) individuals with cognitive impairment that prevents them from understanding the study design and completing the Hospital Anxiety and Depression Scale (HADS) questionnaire (10).

Study design. Patients enrolled in the present study were placed in the following groups ( $n=20$ per group): Pre-surgery group, patients who were recently diagnosed (from 15 to 30 days) with CRC, admitted for tumor resection at the Clinical Hospital of the Faculty of Medicine of Ribeirão Preto; pre-chemotherapy group, patients who underwent surgical resection and who had not commenced adjuvant therapy; chemotherapy group, patients undergoing chemotherapy for $\sim 3$ months (regardless of the regimen adopted for adjuvant treatment); and post-chemotherapy group, patients who completed the adjuvant chemotherapy regimen for $\sim 6$ months. Patients in the prechemotherapy, chemotherapy, and post-chemotherapy groups presented clinical stage III (local tumor in colon or rectum $>5 \mathrm{~cm}$ in diameter and/or has spread to regional lymph nodes) according to staging system of the American Joint Committee on Cancer/Union for International Cancer Control (11).

The control group included 20 healthy volunteers free of any psychiatric or immune system disease. Blood samples were collected from study participants with vacuum tubes (Vacutainer; BD Biosciences, Franklin Lakes, NJ, USA). Depression and anxiety were measured using the Brazilian Portuguese validated version (12) of the HADS (10). The study was conducted in accordance with The Code of Ethics of the World Medical Association (Declaration of Helsinki) and was approved by the Ethics Committee of College of Nursing, University of São Paulo (Ribeirão Preto, SP, Brazil). All patients provided written informed consent.
Psychological assessment. HADS is a brief instrument for measuring psychological distress in cancer patients. It consists of 14 items and contains two subscales, anxiety and depression. Each item is rated on a four-point scale, giving maximum scores of 21 for the two subscales. Scores of 11-21 on each subscale are considered a significant case of psychological morbidity, while scores of 8-10 represent 'borderline', and 0-7 are considered normal (10). For the combined anxiety and depression score (the total HADS score), a cut-off score of 19 was used in order to identify patients with severe affective psychopathology (13).

Cytokine analysis. Venous blood samples $(8 \mathrm{ml})$ were obtained after the application of the questionnaires and were centrifuged at $1,000 \mathrm{x}$ for $10 \mathrm{~min}$ at $4^{\circ} \mathrm{C}$ and the sera were stored at $-80^{\circ} \mathrm{C}$ until assayed. The concentrations of fractalkine were measured using cytometric bead array (CBA) kits (BD Biosciences) according to the manufacturer's instructions. CBA was performed using a BD ${ }^{\mathrm{TM}}$ FACSCanto flow cytometer. Quantitative analysis was performed using FCAP Array $^{\mathrm{TM}}$ v3.0 Software (BD Biosciences).

Statistical analysis. Data were analyzed using GraphPad Prism 5 software (GraphPad Software, Inc., La Jolla, CA, USA). To analyze the hypothesis of equal means between the groups, Kruskal-Wallis one-way ANOVA followed by Dunn's multiple comparison post hoc test were used. The results were expressed as means \pm standard deviation. Pearson's $\chi^{2}$ test was also performed to investigate correlation among the evaluated parameters. Simple linear regression was used to verify the correlation between variables. $\mathrm{P}<0.05$ was considered to indicate a statistically significant difference.

\section{Results}

Demographic characteristics. The characteristics of the study participants are presented in Table I. Twenty healthy volunteers formed the control group. The mean age was $47.8 \pm 9.0$ years and eight $(40 \%)$ were male. The majority were married $(80 \%)$ and had received a secondary education (65\%). The pre-surgery group consisted of patients with $\mathrm{CRC}$ who did not undergo surgical resection. The mean age was $63 \pm 11.8$ years, nine $(45 \%)$ were male, the majority were married $(80 \%)$, and had completed primary education $(65 \%)$. Patients who underwent surgical resection and who did not start adjuvant therapy formed the pre-chemotherapy group (mean age, $56.8 \pm 7.2$ years). Among the 20 subjects in the group, nine $(45 \%)$ were male, married, and had completed secondary education. Patients undergoing chemotherapy for $\sim 3$ months formed the chemotherapy group, with mean age of $56.4 \pm 8.6$ years. Twelve $(60 \%)$ were married, $10(50 \%)$ were male and had completed secondary education. The post-chemotherapy group consisted of patients who had completed an adjuvant chemotherapy regimen for $\sim 6$ months. The mean age was 59.9 \pm 8.7 years, $10(50 \%)$ were male and married and nine (45\%) had completed primary education. No significant differences regarding age, sex, marital status or education level were identified between the groups.

HADS score. Clinically significant levels of anxiety or depression (indicated by respective HADS scores between 11 and 21) 
Table I. Demographic characteristics of study participants.

\begin{tabular}{|c|c|c|c|c|c|c|}
\hline Characteristics & $\begin{array}{c}\text { Healthy } \\
\text { volunteers no. (\%) }\end{array}$ & $\begin{array}{l}\text { Pre-surgery, } \\
\text { no. }(\%)\end{array}$ & $\begin{array}{c}\text { Pre-chemotherapy, } \\
\text { no. }(\%)\end{array}$ & $\begin{array}{c}\text { Chemotherapy, } \\
\text { no. }(\%)\end{array}$ & $\begin{array}{c}\text { Post-chemotherapy, } \\
\text { no. }(\%)\end{array}$ & P-value \\
\hline Age $($ mean $\pm S D)$ & $47.8 \pm 9$ & $63 \pm 11.8$ & $56.8 \pm 7.2$ & $56.4 \pm 8.6$ & $59.9 \pm 8.7$ & $\mathrm{~ns}^{\mathrm{a}}$ \\
\hline \multicolumn{7}{|l|}{ Sex } \\
\hline Male & $8(40)$ & $9(45)$ & $9(45)$ & $10(50)$ & $10(50)$ & $\mathrm{ns}^{\mathrm{b}}$ \\
\hline Female & $12(60)$ & $11(55)$ & $11(55)$ & $10(50)$ & $10(50)$ & \\
\hline \multicolumn{7}{|l|}{ Marital status } \\
\hline Single & $2(10)$ & $1(5)$ & $7(35)$ & $7(35)$ & $6(30)$ & $\mathrm{ns}^{\mathrm{b}}$ \\
\hline Married & $16(80)$ & $16(80)$ & $9(45)$ & $12(60)$ & $10(50)$ & \\
\hline Divorced & $1(5)$ & $1(5)$ & - & - & - & \\
\hline Widowed & $1(5)$ & $2(10)$ & $4(20)$ & $1(5)$ & $4(20)$ & \\
\hline \multicolumn{7}{|l|}{ Education level } \\
\hline Primary & $6(30)$ & $13(65)$ & $9(45)$ & $10(50)$ & $8(40)$ & $\mathrm{ns}^{\mathrm{b}}$ \\
\hline Secondary & $13(65)$ & $6(30)$ & $6(30)$ & $8(40)$ & $9(45)$ & \\
\hline College/university & $1(5)$ & $1(5)$ & $5(25)$ & $2(10)$ & $3(15)$ & \\
\hline
\end{tabular}

${ }^{a}$ Kruskal-Wallis post hoc Dunn's test; 'Pearson's $\chi^{2}$ test. SD, standard deviation; ns, not significant.

Table II. Depression and anxiety scores of study participants.

\begin{tabular}{|c|c|c|c|c|c|c|}
\hline Characteristics & $\begin{array}{c}\text { Healthy } \\
\text { volunteers, no. (\%) }\end{array}$ & $\begin{array}{l}\text { Pre-surgery, } \\
\text { no. }(\%)\end{array}$ & $\begin{array}{c}\text { Pre-chemotherapy, } \\
\text { no. }(\%)\end{array}$ & $\begin{array}{c}\text { Chemotherapy, } \\
\text { no. }(\%)\end{array}$ & $\begin{array}{c}\text { Post-chemotherapy, } \\
\text { no. }(\%)\end{array}$ & P-value \\
\hline \multicolumn{7}{|l|}{ Anxiety (HADS) } \\
\hline $0-7$ & $17(85)$ & $3(15)$ & $4(20)$ & $5(25)$ & $6(30)$ & \multirow[t]{4}{*}{$<0.001^{\mathrm{a}}$} \\
\hline $8-10$ & $3(15)$ & $6(30)$ & $4(20)$ & $9(45)$ & $9(45)$ & \\
\hline $11-21$ & $0(0)$ & $11(55)$ & $12(60)$ & $6(30)$ & $5(25)$ & \\
\hline Mean \pm SD & $3.2 \pm 2.7$ & $11 \pm 3.7$ & $11 \pm 3.9$ & $10 \pm 3.0$ & $9.2 \pm 3.3$ & \\
\hline \multicolumn{7}{|c|}{ Depression (HADS) } \\
\hline $0-7$ & $20(100)$ & $3(15)$ & $5(25)$ & $3(15)$ & $3(15)$ & \multirow[t]{4}{*}{$<0.001^{\mathrm{a}}$} \\
\hline $8-10$ & $0(0)$ & $7(35)$ & $9(45)$ & $4(20)$ & $12(60)$ & \\
\hline $11-21$ & $0(0)$ & $10(50)$ & $6(30)$ & $13(65)$ & $5(25)$ & \\
\hline Mean \pm SD & $2.5 \pm 2.3$ & $11.0 \pm 3.4$ & $9.9 \pm 3.7$ & $12.0 \pm 3.2$ & $9.3 \pm 2.9$ & \\
\hline \multicolumn{7}{|c|}{$\begin{array}{l}\text { Depression and anxiety } \\
\text { (total HADS score) }\end{array}$} \\
\hline$\leq 19$ & $20(100)$ & $7(35)$ & $8(40)$ & $8(40)$ & $12(60)$ & \multirow[t]{2}{*}{$<0.001^{\mathrm{a}}$} \\
\hline$>19$ & $0(0)$ & $13(65)$ & $12(60)$ & $12(60)$ & $8(40)$ & \\
\hline
\end{tabular}

HADS, Hospital Anxiety and Depression Scale. ${ }^{a}$ Pearson's $\chi^{2}$ test. SD, standard deviation.

were verified in CRC patients at different stages of antitumor therapy (Table II). Each evaluated group had at least $40 \%$ patients with a severe combination of depression and anxiety (HADS total score, $>19$ ).

Serum levels offractalkine. The fractalkine serum levels were observed to be elevated in CRC patients at the pre-surgery $(\mathrm{P}<0.001)$ and pre-chemotherapy $(\mathrm{P}<0.001)$ stages, but reduced following chemotherapy $(\mathrm{P}<0.05)$ when compared with the healthy group (Fig. 1).
Correlations between serum levels of fractalkine and HADS scores. Fractalkine serum levels positively correlated $(\mathrm{P}<0.05)$ with anxiety and/or depression (HADS scores) in CRC patients at different stages of antitumor therapy (Table III). However, in the post-chemotherapy group, no correlation was identified between fractalkine levels and depression scores. The global correlation between fractalkine levels and HADS scores, regardless of the group, was also quantified, and the highest depression and anxiety scores were associated with the highest fractalkine serum levels. 
Table III. Correlation between serum levels of cytokines and HADS score in each group $(n=20)$.

\begin{tabular}{lccc}
\hline Group & $\begin{array}{c}\text { Anxiety } \\
\text { (HADS) }\end{array}$ & $\begin{array}{c}\text { Depression } \\
\text { (HADS) }\end{array}$ & $\begin{array}{c}\text { Depression } \\
\text { and anxiety } \\
\text { (total HADS) }\end{array}$ \\
\hline Healthy volunteers & 0.16 & 0.18 & 0.16 \\
Pre-surgery & $0.45^{\mathrm{a}}$ & $0.35^{\mathrm{b}}$ & $0.26^{\mathrm{c}}$ \\
Pre-chemotherapy & $0.32^{\mathrm{b}}$ & $0.40^{\mathrm{b}}$ & $0.42^{\mathrm{b}}$ \\
Chemotherapy & $0.40^{\mathrm{b}}$ & $0.24^{\mathrm{c}}$ & $0.38^{\mathrm{b}}$ \\
Post-chemotherapy & $0.25^{\mathrm{c}}$ & 0.16 & $0.26^{\mathrm{c}}$ \\
\hline
\end{tabular}

${ }^{\mathrm{a}} \mathrm{P}<0.0001,{ }^{\mathrm{b}} \mathrm{P}<0.001,{ }^{\mathrm{c}} \mathrm{P}<0.05$ (simple linear regression). HADS, Hospital Anxiety and Depression Scale.

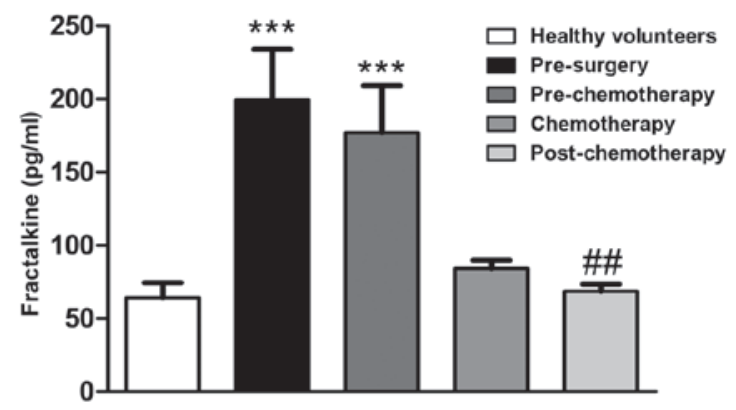

Figure 1. Fractalkine serum levels in colorectal cancer patients at different stages of antitumor therapy. Fractalkine levels were measured by cytometric bead array (CBA). Data represent the mean \pm standard error of the mean from a CBA assay of blood samples. ${ }^{* * * *} \mathrm{P}<0.0001$ vs. healthy volunteers group; ${ }^{\# \#} \mathrm{P}<0.001$ vs. pre-chemotherapy group.

\section{Discussion}

In the current study, the possible correlation of fractalkine with anxiety and depression symptoms was analyzed in CRC patients. Elevated fractalkine serum levels were identified in CRC patients, which varied between the different stages of antitumor therapy, and positive correlations were observed between fractalkine levels and depression and anxiety scores. Therefore, it was hypothesized that fractalkine is involved in such mood disorders that are observed in CRC patients.

Although various mechanisms act on the regulation of fractalkine expression in different types of cells, a major mechanism seems to involve the activation of the nuclear factor (NF)- $\kappa \mathrm{B}$ signaling pathway by tumor necrosis factor (TNF)- $\alpha$ that may occur on tumor cells allowing the release of the chemokine in the tumor microenvironment and its reach to the bloodstream (14). Indeed, the level of NF- $\kappa \mathrm{B}$ expression has been shown to be increased in many types of tumors, including CRC (15) and elevated TNF- $\alpha$ levels have been identified in CRC patients (16). Thus, increased expression levels of fractalkine may be a consequence of antitumor immune responses, which in turn triggers systemic effects that include those in the central nervous system, which are emphasized in the present study.

The role of fractalkine in the regulation of the immune response in the brain has been extensively investigated. This chemokine is involved in microglial activation by neurons and the local expression of this mediator enables the modulation of inflammatory activity in the central nervous system (17). However, fractalkine participates in the normal physiology of neuronal cells with a relevant role in neurodegenerative diseases and impaired recovery from sickness behavior (18). Furthermore, studies using animal models have described the effects of fractalkine on brain physiology, which are associated with depression and anxiety (6). Mice deficient in CX3CR1 cells have been shown to exhibit resistance to stress-induced depression-like behavior and to not respond to antidepressant treatment (19). Therefore, high levels of systemic fractalkine seems to be linked to the incidence of depression and anxiety.

In the current study, pre-surgery CRC patients (who had not begun chemotherapy) exhibited the highest levels of depression and anxiety symptoms, which may be attributed to the fact that these patients must deal with the fear, frustration and uncertainty of successful treatment. These patients also presented the highest serum levels of fractalkine and the highest level of agreement in the correlation analysis. Such a correlation is not arbitrary, and the current study hypothesized that the fractalkine secreted in the tumor environment reaches the brain, via the circulation, affecting areas responsible for anxious/depressive behavior. However, patients in the final stages of treatment, who underwent surgical resection of the tumor, exhibited depression and anxiety symptoms, and fractalkine levels similar to those of healthy volunteers, which indicates that the tumor and other cells in the tumor microenvironment are a major source of serum fractalkine in CRC patients.

Based on the current results, it is recommended that all CRC patients be routinely screened for psychological distress, particularly if they exhibit increased serum levels of fractalkine. Routine dosage of this serum chemokine may serve as a biomarker for the manifestation of depression and anxiety in cancer patients, for diagnosis and monitoring the patient. Thus, an integral understanding of the mechanisms linking fractalkine to mood disorders will allow the design of interventions that lead to an improved quality of life and overall survival of CRC patients.

Limitations of this study design include its cross-sectional nature and the relatively small number of colorectal patients that were included. Therefore, further studies are required to confirm the current results. In conclusion, these preliminary findings indicate that fractalkine may serve as a useful clinical biomarker of depression and/or anxiety risk among CRC patients undergoing antitumor therapy. Furthermore, as fractalkine is potentially involved in the pathophysiology of these comorbidities, it is proposed that fractalkine is included in the routine screening of CRC patients to allow for psychological intervention to oncological patients in high-risk situations.

\section{Acknowledgements}

The present study was supported by a grant for São Paulo Research Foundation (FAPESP) (grant nos. 2011/17118-9 and 2013/01262-9). The authors would like to thank the staff of the Service of Clinical Oncology of Clinical Hospital of the Faculty of Medicine of Ribeirão Preto (Ribeirão Preto, SP, 
Brazil), for assistance with participant recruitment, and all the participants who generously gave their time to take part.

\section{References}

1. Marchesi F, Locatelli M, Solinas G, Erreni M, Allavena P and Mantovani A: Role of CX3CR1/CX3CL1 axis in primary and secondary involvement of the nervous system by cancer. J Neuroimmunol 224: 39-44, 2010.

2. Nelson PJ and Muenchmeier N: Membrane-anchored chemokine fusion proteins: A novel class of adjuvants for immunotherapy. OncoImmunology 2: e26619, 2013.

3. Yao X, Qi L, Chen X, Du J, Zhang Z and Liu S: Expression of CX3CR1 associates with cellular migration, metastasis, and prognosis in human clear cell renal cell carcinoma. Urol Oncol 32: 162-170, 2014.

4. Wada A, Ito A, Iitsuka H, Tsuneyama K, Miyazono T, Murakami J, Shibahara N, Sakurai H, Saiki I, Nakayama T, et al: Role of chemokine CX3CL1 in progression of multiple myeloma via CX3CR1 in bone microenvironments. Oncol Rep 33: 2935-2939, 2015.

5. Agalliu I, Xue X, Cushman M, Cornell E, Hsing AW, Kaplan RC, Anastos K, Rajpathak S and Ho GY: Detectability and reproducibility of plasma levels of chemokines and soluble receptors Results Immunol 3: 79-84, 2013.

6. Rogers JT, Morganti JM, Bachstetter AD, Hudson CE, Peters MM Grimmig BA, Weeber EJ, Bickford PC and Gemma C: CX3CR1 deficiency leads to impairment of hippocampal cognitive function and synaptic plasticity. J Neurosci 31: 16241-16250, 2011.

7. Dunn J, Lynch B, Rinaldis M, Pakenham K, McPherson L, Owen N, Leggett B, Newman B and Aitken J: Dimensions of quality of life and psychosocial variables most salient to colorectal cancer patients. Psychooncology 15: 20-30, 2006.

8. Russell L, Gough K, Drosdowsky A, Schofield P, Aranda S, Butow PN, Westwood JA, Krishnasamy M, Young JM, Phipps-Nelson J, et al: Psychological distress, quality of life, symptoms and unmet needs of colorectal cancer survivors near the end of treatment. J Cancer Surviv 9: 462-470, 2015.

9. Grivennikov SI and Karin M: Inflammatory cytokines in cancer: Tumour necrosis factor and interleukin 6 take the stage. Ann Rheum Dis 70 (Suppl 1): i104-i108, 2011.
10. Zigmond AS and Snaith RP: The hospital anxiety and depression scale. Acta Psychiatr Scand 67: 361-370, 1983.

11. American Joint Committee on Cancer (AJCC): AJCC Cancer Staging Manual. Edge, SB, Byrd, DR, Compton, CC, Fritz AG, Greene, FL and Trotti A (eds). 7th edition. Springer, New York, NY, p143, 2010

12. Botega NJ, Bio MR, Zomignani MA, Garcia C Jr and Pereira WA: Mood disorders among inpatients in ambulatory and validation of the anxiety and depression scale HAD. Rev Saude Publica 29: 355-363, 1995 (In Portuguese).

13. Razavi D, Delvaux N, Farvacques C and Robaye E: Screening for adjustment disorders and major depressive disorders in cancer in-patients. Br J Psychiatry 156: 79-83, 1990.

14. Isozaki T, Kasama T, Takahashi R, Odai T, Wakabayashi K, Kanemitsu H, Nohtomi K, Takeuchi HT, Matsukura S and Tezuka M: Synergistic induction of CX3CL1 by TNF alpha and IFN gamma in osteoblasts from rheumatoid arthritis: Involvement of NF-kappa B and STAT-1 signaling pathways. J Inflamm Res 1: 19-28, 2008.

15. Wang S, Liu Z, Wang L and Zhang X: NF-kappaB signaling pathway, inflammation and colorectal cancer. Cell Mol Immunol 6: 327-334, 2009.

16. Oliveira Miranda D, Soares de Lima TA, Ribeiro Azevedo L, Feres O, Ribeiro da Rocha JJ and Pereira-da-Silva G: Proinflammatory cytokines correlate with depression and anxiety in colorectal cancer patients. BioMed Res Int 2014: 739650, 2014.

17. Zujovic V, Schussler N, Jourdain D, Duverger D and Taupin V: In vivo neutralization of endogenous brain fractalkine increases hippocampal TNFalpha and 8-isoprostane production induced by intracerebroventricular injection of LPS. J Neuroimmunol 115 135-143, 2001

18. Briones TL, Woods J and Wadowska M: Retraction Note: Chronic neuroinflammation and cognitive impairment following transient global cerebral ischemia: role of fractalkine/CX3CR1 signaling. J Neuroinflammation 12: 220, 2015.

19. Hellwig S, Brioschi S, Dieni S, Frings L, Masuch A, Blank T and Biber K: Altered microglia morphology and higher resilience to stress-induced depression-like behavior in CX3CR1-deficient mice. Brain Behav Immun 55: 126-137, 2016. 